Jour. of inverse and ill-posed problems, 4, N6, (1996), 531-534.

\title{
MINIMIZATION OF THE TOTAL RADIATION \\ FROM AN OBSTACLE BY A CONTROL FUNCTION ON A PART OF ITS BOUNDARY
}

\author{
A.G. RAMM \\ Department of Mathematics, Kansas State University, Manhattan, KS 66506-2602 \\ ABstract. It is proved that by choosing a boundary control function on an arbitrarily \\ small open subset of the boundary one can reduce the total radiation from an obstacle at \\ a fixed direction of the incident wave and fixed frequency to arbitrarily small level
}

\section{Introduction.}

In this paper the following question is answered. Suppose one has an obstacle, that is a bounded body which scatters waves. A plane wave with a fixed direction of propagation, $\alpha$ and a fixed wavenumber $k$ is incident upon the obstacle. On an arbitrary small part of the boundary of the obstacle one can have a control function. Can one choose this control function so that the total radiation from the obstacle becomes as small as one wishes? The answer is yes, and we prove this in the paper. Consider the obstacle scattering problem:

$$
\begin{gathered}
\nabla^{2} u+k^{2} u=0 \text { in } D^{\prime}, \\
u_{N}=0 \text { on } \Gamma_{1}, \quad v_{N}=w, \text { on } \Gamma_{2}, \\
u=u_{0}+v, \quad u_{0}:=\exp (i k \alpha \cdot x),
\end{gathered}
$$

where $D^{\prime}:=\mathbb{R}^{3} \backslash D, \quad D$ is a bounded domain with a smooth boundary $\Gamma, k>0$ is a fixed wavenumber, $\alpha \in S^{2}$ is a given unit vector, $S^{2}$ is the unit sphere, $v$ satisfies the radiation condition

$$
\lim _{r \rightarrow \infty} \int_{|x|=r}\left|v_{r}-i k v\right|^{2} d s=0
$$

$N$ is the exterior unit normal to $\Gamma, \Gamma_{1} \cup \Gamma_{2}=\Gamma, \Gamma_{j}, j=1,2$, are parts of the boundary which do not have common open subsets, and we assume that $\Gamma_{2}$ is an open subset in $\Gamma$. The boundary control function $w$ is at our disposal. We assume that the boundary is sufficiently smooth for the uniqueness of the solution to the Cauchy problem for the Helmholtz equation to hold. The above problem has a unique solution [1]. The function $v$ has the following asymptotics

$$
v=A\left(\alpha^{\prime}, \alpha, k\right) \frac{\exp (i k r)}{r}+o\left(\frac{1}{r}\right) \text { as } r \rightarrow \infty, \alpha^{\prime}:=\frac{x}{r} .
$$

The coefficient $A\left(\alpha^{\prime}, \alpha, k\right)$ is called the scattering amplitude. Define the total crossection:

$$
\sigma:=\int_{S^{2}}\left|A\left(\alpha^{\prime}, \alpha, k\right)\right|^{2} d \alpha^{\prime} .
$$

1991 Mathematics Subject Classification. 35R30.

Key words and phrases. scattering, boundary control.

The author thanks UJF for hospitality, Dr.J.Jaffre for the opportunity to visit INRIA-Rocquencourt, and Dr J.D.Bonamou for a discussion in which the problem solved in this note was formulated. 
We fix $\alpha \in S^{2}$ and $k>0$. The problem we study is:

can $w \in L^{2}\left(\Gamma_{2}\right)$ be chosen so that $\sigma$ becomes as small as we wish?

If yes, then we can say that the obstacle can be made invisible for the incident wave coming from the direction $\alpha$ at the frequency $k$.

Our basic result is an affirmative answer to the above question. This answer is formulated in Theorem 1.

Theorem 1. $\inf \sigma=0$, where the infimum is taken over all $w \in C_{0}^{\infty}\left(\Gamma_{2}\right)$.

Note that the infimum is not attained. A proof of this result is given in section 2. Our technique is applicable to other boundary conditions, the Dirichlet and Robin conditions in particular. The case of Lipschitz boundaries for the scattering problems is treated in [3] and [4].

\section{Proofs.}

We assumed that $n=3$. The proof is essentially the same for any $n \geq 2$.

Proof. Let us outline the ideas. Let

$$
\left(\nabla^{2}+k^{2}\right) g=-\delta(x-y) \text { in } D^{\prime}, \quad g_{N}=0 \text { on } \Gamma,
$$

and $g$ satisfies the radiation condition. Using Green's formula, one gets:

$$
v(x)=-\int_{\Gamma} g v_{N} d s=-\int_{\Gamma_{2}} g w d s+\int_{\Gamma_{1}} g u_{0 N} d s .
$$

Let us drop $k$-dependence since $k$ is fixed. It is proved in $[1, \mathrm{p} .46]$ that

$$
g(x, y)=\frac{\exp (i k r)}{4 \pi r} \phi(y, \alpha, k)+o\left(\frac{1}{r}\right), \quad r \rightarrow \infty, r:=|x|, \frac{x}{r}=-\alpha,
$$

where $\phi$ is the scattering solution: it satisfies equation (1), the Neumann boundary condition on $\Gamma$, and has representation (3) with $v$ satisfying the radiation condition.

From (6) and (7) one gets:

$$
A\left(\alpha^{\prime}, \alpha\right)=-\frac{1}{4 \pi} \int_{\Gamma_{2}} \phi\left(s,-\alpha^{\prime}\right) w d s+\nu,
$$

where

$$
\nu:=\frac{1}{4 \pi} \int_{\Gamma_{1}} \phi\left(s,-\alpha^{\prime}\right) u_{0 N} d s, \quad \nu \in L^{2}\left(S^{2}\right) .
$$

The conclusion of Theorem 1 follows immediately from Lemma 1 which is stated and proved below. Indeed, if Lemma 1 holds, then one can choose $w$ to make $L^{2}\left(S^{2}\right)$-norm of the scattering amplitude $A\left(\alpha^{\prime}, \alpha\right)$ as small as one wishes, which is the same as making $\sigma$ in (5) as small as one wishes.

Lemma 1. The set $\left\{\int_{\Gamma_{2}} \phi(s, \alpha) w d s\right\} \quad \forall w \in C_{0}^{\infty}\left(\Gamma_{2}\right)$ is complete in $L^{2}\left(S^{2}\right)$.

Proof. The ideas of the proof are related to the ideas introduced in [2]. If Lemma 1 is false, then there exists an $f \in L^{2}\left(S^{2}\right)$ such that

$$
0=\int_{S^{2}} d \alpha f(\alpha) \phi(s, \alpha) \quad \forall s \in \Gamma_{2} .
$$


Denote

$$
h(x):=\int_{S^{2}} d \alpha f(\alpha) \phi(x, \alpha) .
$$

Then

$$
\left(\nabla^{2}+k^{2}\right) h=0 \text { in } D^{\prime}, h_{N}=0 \text { on } \Gamma, h=0 \text { on } \Gamma_{2} .
$$

This and uniqueness of the solution to the Cauchy problem imply that $h=0$ in $D^{\prime}$ :

$$
h(x)=\int_{S^{2}} d \alpha f(\alpha) \phi(x, \alpha)=0 \quad \forall x \in D^{\prime}
$$

We derive from (13) that

$$
h_{0}(x):=\int_{S^{2}} d \alpha f(\alpha) \exp (i k \alpha \cdot x)=0 \quad \forall x \in D^{\prime}
$$

Since $h_{0}(x)$ is an entire function of $x$, equation (14) implies that $h_{0}(x)=0$ in $\mathbb{R}^{3}$, and, by the injectivity of the Fourier transform, we conclude that $f(\alpha)=0$. The proof of Lemma 1 is therefore complete as soon as we derive (14) from (13). This derivation goes as follows.

Define

$$
B \phi:=\phi(x, \alpha)-\int_{\Gamma} G_{N}(x, s) \phi(s, \alpha) d s,
$$

where $G:=\frac{\exp (i k|x-y|}{4 \pi|x-y|}$

By Green's formula one gets:

$$
\phi(x, \alpha)=u_{0}(x, \alpha)+\int_{\Gamma} G_{N}(x, s) \phi(s, \alpha) d s
$$

so that

$$
B \phi=u_{0}(x, \alpha) .
$$

Since $B$ acts on the $x$-variable only, one can apply $B$ to $h(x)$ in (13) and get (14). The proof of Lemma 1 is complete.

This completes the proof of Theorem 1.

\section{REFERENCES}

1. A.G.Ramm, Scattering by obstacles, Reidel, Dordrecht, 1986.

2. A.G.Ramm, Multidimensional inverse scattering problems, Longman/Wiley, New York, 1992.

3. A.G.Ramm, Uniqueness theorems for inverse obstacle scattering problems in Lipschitz domains, Applic. Analysis 59 (1995), 377-383.

4. A.G.Ramm and A.Ruiz, Existence and uniqueness of scattering solutions in non-smooth domains, J. Math. Anal. Appl 201 (1996), 329-338.

e-mail: ramm@math.ksu.edu 